\title{
Análisis de las prestaciones de un motor de combustión interna alternativo usando biocombustibles: (i) aceite de soya; (ii) aceite de canola
}

\section{Analysis of performance of a reciprocating internal combustion engines using biofuels: (i) soybean oil; (ii) canola oil}

\author{
JIMÉNEZ-MACEDO, Víctor Daniel†*1, VILLAGÓMEZ-GALINDO, Miguel ${ }^{1}$ y DE LA GARZA-DE
} LEÓN, Oscar Alejandro ${ }^{2}$

${ }^{1}$ Universidad Michoacana de San Nicolás de Hidalgo, Facultad de Ingeniería Mecánica

${ }^{2}$ Universidad Autónoma de Nuevo León

ID $1^{\text {er }}$ Autor: Víctor Daniel Jiménez Macedo / ORC ID: 0000-0001-5199-0698, CVU CONACYT ID: 240808

ID $1^{\text {er }}$ Coautor: Miguel Villagómez Galindo / ORC ID: 0000-0002-4560-2529, CVU CONACYT ID: 354258

ID $2^{\text {do }}$ Coautor: Oscar Alejandro, de la Garza-De León / ORC ID: 0000-0001-7528-3322, CVU CONACYT ID: 220054

DOI: $10.35429 / J R E .2020 .13 .4 .9 .17$

Recibido 3 de Junio, 2020; Aceptado Octubre 30, 2020

\section{Resumen}

En el presente estudio se evalúan dos biocombustibles derivado de aceite de cocina. Para realizar el presente trabajo se usará un motor de combustión interna alternativo de encendido por compresión, que está dispuesto en un banco de pruebas motor completamente instrumentado. Primeramente, se describe el proceso de elaboración del biodiésel, donde se ha usado aceite de soya y aceite canola. Posteriormente, se describe el apartado experimental que se ha usado para la presente investigación. Los ensayos experimentales se realizan: (i) modificando el grado de apertura del acelerador y (ii) usando una carga constante del freno-motor. Seguidamente, en la sección de resultados se cuantifica el desempeño de los dos biocombustibles generados frente al diésel de uso comercial. También, se muestran las diferencias porcentuales de: (i) potencia motor; (ii) gasto másico de combustible; (iii) eficiencia térmica del motor térmico y; (iv) temperaturas de escape. Los ensayos se han realizado bajo diferentes condiciones de régimen de giro del motor de combustión interna alternativo. Donde las diferencias promedio principales que se han registrado en banco motor fueron: para la potencia, del 32 y $33 \%$; para el gasto másico del combustible del -4.5 y $5 \%$, cuando se usa el biodiésel de soya y el biodiésel de canola, respectivamente. Estas diferencias se deben al contenido energético de los biocombustibles y a la forma en que se lleva a cabo el proceso de combustión en el interior del motor de combustión interna.

Motor de combustión interna, Biodiésel, Emisiones contaminantes

\begin{abstract}
In the present study, two biofuels derived from cooking oil are evaluated. To carry out this work, an reciprocating internal combustion engine will be used, which is ready on a fully instrumented engine test bench. Firstly, the biodiesel production process is described, where soybean oil and canola oil have been used. Subsequently, the experimental section that has been used for the present investigation is described. The experimental tests are carried out: (i) modifying the degree of opening of the accelerator and (ii) using a constant load of the dynamometer. On the other hand, in the results section shows the performance of the two biofuels generated against diesel commercial. Also, in this section shows the percentage differences of: (i) engine power; (ii) mass fuel, (iii) thermal efficiency engine and, (iv) exhaust temperaturas. The tests have been carried out under different engine speed conditions of the reciprocating internal combustion engine. Where, the main average differences that have been registered in the engine test bench were: for engine power, 32 and 33\%; for the mass fuel consumption of -4.5 and 5\%, when using soybean biodiesel and canola biodiesel, respectively. These differences are due to the energy capacity of each biofuel and, the instantaneous evolution of combustion process inside the reciprocating internal combustion engine.
\end{abstract}

Reciprocating internal combustión engine, Biodiesel, Polluting emissions

Citación: JIMÉNEZ-MACEDO, Víctor Daniel, VILLAGÓMEZ-GALINDO, Miguel y DE LA GARZA-DE LEÓN, Oscar Alejandro. Análisis de las prestaciones de un motor de combustión interna alternativo usando biocombustibles: (i) aceite de soya; (ii) aceite de canola. Revista de Energías Renovables. 2020. 4-13: 9-17

*Correspondencia al Autor (correo electrónico: vdjimen@umich.mx)

$\dagger$ Investigador contribuyendo como primer autor. 


\section{Introducción}

Los biocombustibles juegan un papel importante dentro del contexto del uso de combustibles derivados del petróleo. Principalmente, porque estos últimos son perecederos $\mathrm{y}$, también, por el constante intento de reducir las emisiones que se generan durante el proceso de combustión en el interior de los motores de combustión interna alternativos. El biodiesel es un combustible de características químicas y térmicas similares al diésel de uso comercial.

Entre las principales ventajas del biodiésel son; su bajo contenido de azufre, compuestos aromáticos, reducción de emisiones de CO y CO2, como lo indica Peyton (2016). De esta forma, se reduce la contaminación que provoca el efecto invernadero y la lluvia ácida. Para la producción de biodiésel, las principales materias primas son la grasa animal, aceite de cocina usado, habas de soya y jatropha, como lo indica Mofijur (2016). De esta forma es posible reducir la dependencia, preservando el petróleo, así como, restaurar la lubricidad del diésel de bajo contenido de azufre, agregando niveles bajos de 1-2 \% de biodiésel como lo muestra Gerhard Knothe (2005).

También, la generación de nuevos combustibles puede proporcionar mejores rendimientos en el motor de combustión interna alternativo. No obstante, otras soluciones, como el uso de regeneradores, son desarrolladas para mejorar el rendimiento del motor como lo propone Stikhun, A. (2020). En este mismo contexto, el empleo de modelos numéricos de simulación por ordenador, permite predecir mejoras en el rediseño de los elementos constructivos del motor térmico, así como, la puesta a punto, como lo realiza Blair G.P. (1996), Payri F. y Desantes J.M. (2011) y García Martínez, E. (2020).

Por otra parte, se observa una concordancia entre la disponibilidad de materia prima y la publicación de artículos científicos ya que en cada país investiga principalmente sobre la materia prima que tiene disponible. También, un factor importante es la disponibilidad de tierras de cultivo, donde el biodiésel que se obtiene con la jatropha tiene una buena primacía, ya que esta materia prima es adaptable a terrenos marginales infructuosos, por lo que no es un riesgo para cultivos alimenticios.
Además, las emisiones que son generadas por los procesos de combustión, que se producen por los motores térmicos, se van hacia la atmósfera. Una vez allí, estos gases contaminantes evolucionan al mezclarse con otras sustancias, dando como resultado un compuesto corrosivo. Estos compuestos ácidos son absorbidos por el suelo en forma de lluvia ácida. Afectando la vegetación, por ejemplo, las hojas, donde el proceso directo es la fotosíntesis. También, la lluvia ácida, tienen efectos adversos en la salud de los árboles, haciéndolos más vulnerables a parásitos. También, esta lluvia ácida deteriora diversos tipos de infraestructura, como, estructuras metálicas o edificios.

Por otra parte, el efecto invernadero, que es motivado en parte por el dióxido de carbono presente en la atmósfera, es deseable en ciertas cantidades, para mantener una temperatura óptima en el planeta. No obstante, la sobrecarga de $\mathrm{CO} 2$ en la atmósfera, genera efectos indeseables, principalmente por el incremento en la temperatura promedio del planeta. Que se debe especialmente a que estos gases retienen, de forma parcial, la radiación térmica que emite la superficie terrestre.

No obstante, por lo dictado previamente, también la capa de $\mathrm{CO} 2$ representa una capa que nos protege de forma parcial, de radiaciones de longitud de onda larga o radiación infrarroja. Los combustibles que generan este tipo de gas contaminante, de mayor a menor contribución, son: el carbón mineral, coque de petróleo, los neumáticos, el diésel, el queroseno, la gasolina, el propano y el gas natural.

\section{Proceso de obtención del biodiésel}

El biodiésel se puede obtener a partir de grasa animal y aceite vegetal de cocina usado, está formado por ésteres monoalquílicos de ácidos grasos. A partir de los esteres metílicos derivados de los ácidos grasos se pueden obtener propiedades fisicoquímicas parecidas a las del diésel de uso comercial.

Esto representa una ventaja, sobre todo cuando se lleva a cabo el proceso de combustión en el interior del motor de combustión interna. No obstante propiedades como la viscosidad y el poder calorífico del combustible son sensibles con la rapidez de quemado y presión en la cámara de combustión respectivamente. 
La obtención del biodiesel, para el presente trabajo, se lleva a cabo mediante el proceso de la transesterificación. Se han obtenido dos biocombustibles, el primero, a partir de aceite de soya y, el segundo, de aceite de canola, que son ampliamente usados en restaurantes y en la cocina de los hogares.

El proceso de la transesterificación se lleva por medio de tres reacciones, primeramente, el triglicérido reacciona con un catalizador, para dar como resultado un diglicérido. Luego, en una segunda reacción, el diglicérido evoluciona dando como resultado un monoglicérido.

Finalmente, se produce un equilibrio químico, con una tercera reacción química, que da como resultado, por una parte, un mol de glicerina $\mathrm{y}$, por otra parte, tres moles de éster alquílico, que para el presente caso es un ácido graso del tipo éster metílico. Una representación del proceso de la transesterificación se muestra en la figura 1 siguiente.

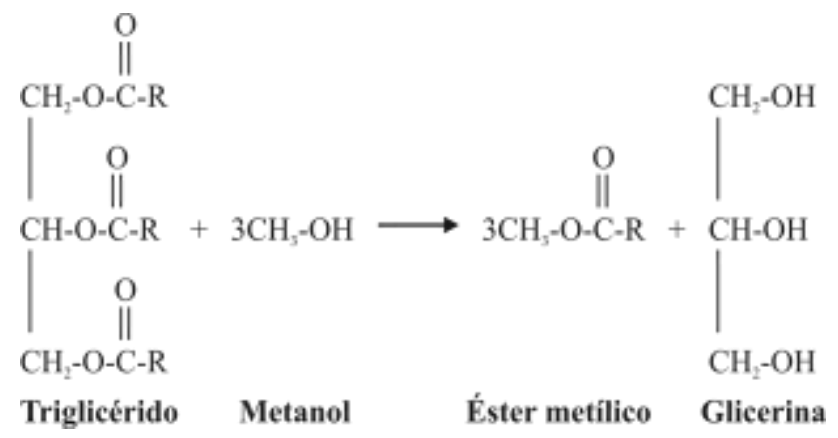

Figura 1 Proceso de transesterificación

En esta figura 1 se muestra que un mol de triglicérido se mezcla con tres moles de alcohol metílico generando una serie de reacciones químicas, que dan como resultado tres moles de éster metílico y un mol de glicerina. Una vez explicado el proceso para la obtención del biocombustible, en la figura 2 se muestra el biodiésel obtenido a partir de aceite de soya.

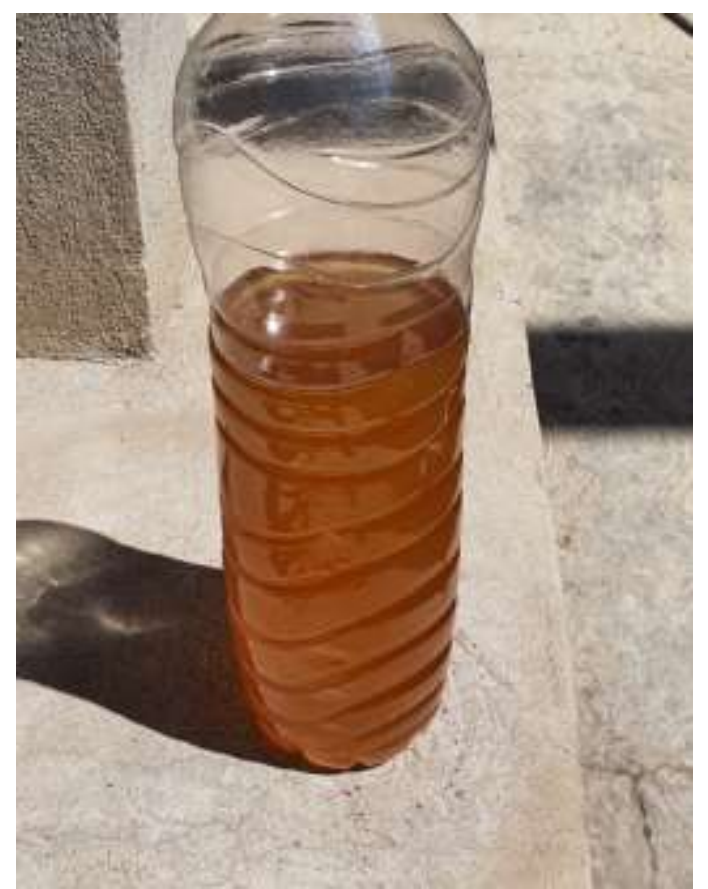

Figura 2 Biodiésel obtenido para su uso en motores de combustión interna alternativos

El biodiésel que se muestra en la figura 2, junto con el biocombustible obtenido mediante el aceite de canola, será usado en el motor de combustión interna alternativo, con el objetivo de realizar una evaluación comparativa de las prestaciones obtenidas de este combustible frente al diésel de uso comercial. No obstante, la caracterización fisicoquímica de los combustibles queda fuera del alcance del presente trabajo.

\section{Apartado experimental}

En la presente sección se detalla los recursos experimentales que se han usado para obtener las prestaciones del motor térmico. El apartado experimental representa un papel importante para cuantificar de forma real los parámetros de medida en los motores de combustión interna. En la figura 3 se muestra el motor de combustión interna que incluye todos sus sistemas que está dispuesto en un banco motor y acoplado a un freno-motor. 


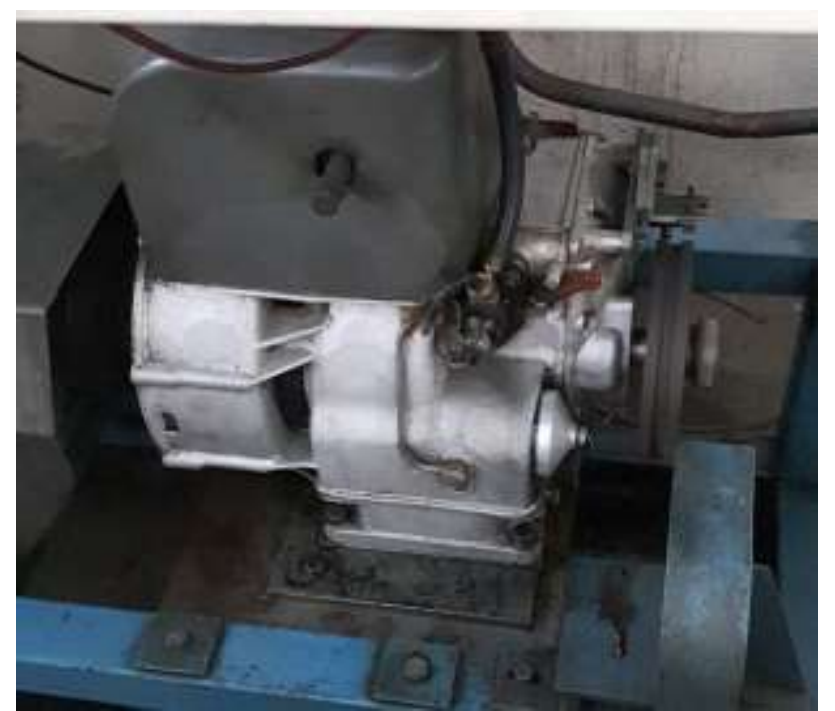

Figura 3 Motor de combustión interna usado en el presente estudio

En esta figura 3 se muestra el motor térmico que incorpora su sistema de admisión y escape. En la siguiente tabla 1, se muestran las principales características geométricas del motor.

\begin{tabular}{ll}
\hline Característica del MCIA & \\
\hline Cilindrada & $219 \mathrm{cc}$ \\
Número de cilindros & Monocilíndrico \\
Diámetro & $69,85 \mathrm{~mm}$ \\
Carrera & $57,15 \mathrm{~mm}$ \\
Válvula de admisión & 1 \\
Válvula de escape & 1 \\
Suministro de combustible & Sistema de inyección \\
\hline
\end{tabular}

Tabla 1 Características geométricas del motor de combustión interna alternativo

De esta forma, en el presente trabajo, se usa un motor de encendido por compresión monocilíndrico, atmosférico y refrigerado por aire. Por otra parte, una vez presentadas las características principales del motor térmico, a continuación, se muestra, en la figura 4, el banco de pruebas motor.

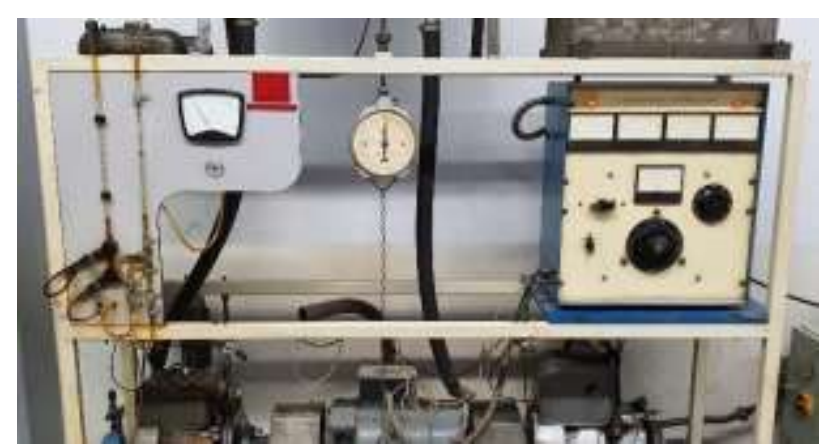

Figura 4 Banco de ensayos motor empleado en el presente trabajo de investigación
El banco de ensayos motor, alberga el motor presentado en la figura 3. Que sirve para medir la potencia del motor térmico, el banco motor permite imponer diferentes condiciones de carga, por medio de un freno-motor. También, para medir el par, el banco incorpora un dinamómetro calibrado. Para la medición del consumo de combustible, el banco tiene instaladas probetas calibradas, para medir la temperatura del gas de escape, se han insertado termopares tipo $\mathrm{K}$, que proporciona información de la transferencia de calor, no obstante, ese estudio queda fuera del alcance del presente trabajo.

También, para medir el régimen de giro del motor, el banco motor tiene instrumentado un tacómetro. De esta forma, las variables que se registran en banco motor son la potencia-motor, el gasto másico de combustible, el régimen de giro motor, el rendimiento térmico del motor y la temperatura de escape. Los ensayos experimentales se han logrado imponiendo diferentes condiciones operativas del motor.

Es de conocimiento común que la potencia que se genera por el motor es el resultado del trabajo realizado en su interior, por el cilindro-pistón, en ciclo cerrado durante la carrera de combustión. Que está en función de la cantidad másica capturada de aire-combustible, en el interior del cilindro, durante el proceso de admisión. En la figura 5 se muestra la potencia desarrollada por el motor de combustión interna, considerando diferentes grados de carga a diferentes regímenes de giro.

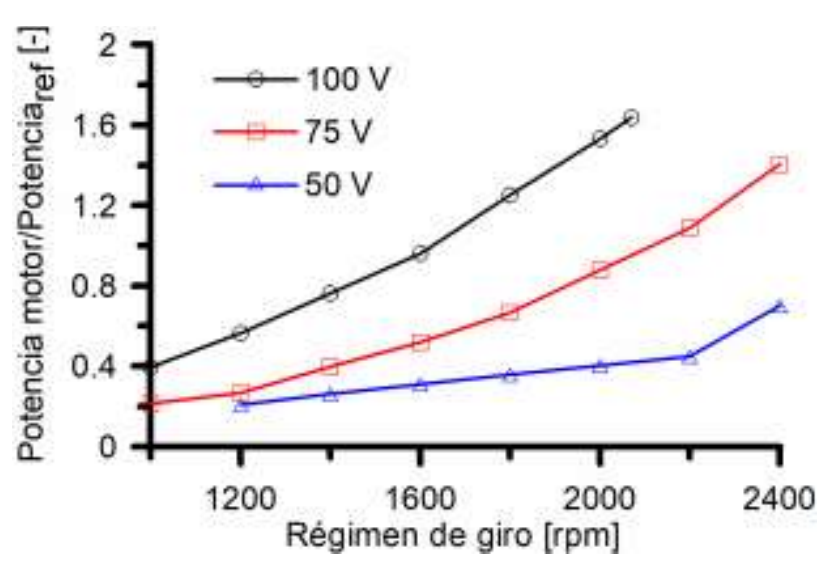

Figura 5 Potencia motor frente al régimen de giro

En esta figura 5 se muestra los resultados obtenidos de potencia, que se muestra está en su forma adimensional, donde se ha usado una condición real ensayada de referencia.

JIMÉNEZ-MACEDO, Víctor Daniel, VILLAGÓMEZ-GALINDO, Miguel y DE LA GARZA-DE LEÓN, Oscar Alejandro. Análisis de las prestaciones de un motor de combustión interna alternativo usando biocombustibles: (i) aceite de soya; (ii) aceite de canola. Revista de Energías Renovables. 2020 
En los resultados obtenidos en la figura 5 , el combustible empleado es el diésel comercial. En esta figura, se muestra que, cuando el motor se somete a una carga o frenomotor mayor, se requiere mayor mezcla de airecombustible, por ende, se genera más potencia para soportar la mayor carga que se induce al motor térmico.

\section{Resultados}

Las prestaciones obtenidas de motor de combustión interna se muestran en esta sección. En la figura 6 se muestra la potencia obtenida con el diésel comercial frente al biodiésel obtenido con aceite de soya.
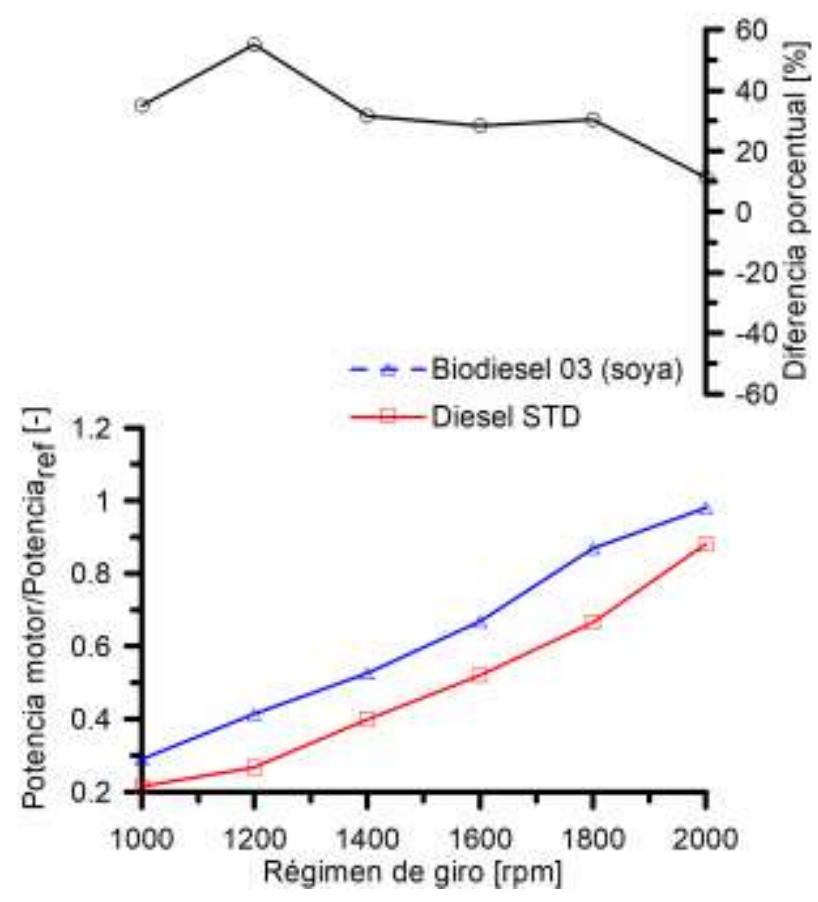

Figura 6 Resultados del banco de ensayos motor. Abajo: potencia adimensional frente al régimen de giro motor; Arriba: la diferencia porcentual de la potencia del motor térmico usando diésel estándar y el biocombustible generado a partir de aceite de soya

En la parte de debajo de la figura 6 se muestra la potencia obtenida del banco de pruebas motor. Los resultados de potencia, en esta figura, se muestra en su forma adimensional, que es de gran utilidad cuando se desea contrastar de forma comparativa. Para ello, fue usada una condición real medida como parámetro de referencia. De esta forma, se aprecia que la potencia registrada, cuando se usa el biocombustible a partir de aceite vegetal de soya es mayor en todas las condiciones de operación.
Por otra parte, en los resultados de diferencia porcentual, que se muestran en la parte de arriba de esta misma figura 5 , se muestran una diferencia máxima de $55 \%$ cuando el tacómetro marca $1200 \mathrm{rpm}$ y; una diferencia mínima de $11 \%$ para un régimen de giro de $2000 \mathrm{rpm}$.

Por otra parte, el consumo de combustible, a una determinada carga del frenomotor, es una variable de interés que puede indicar de forma indirecta el poder calorífico específico del combustible. De esta forma, en la figura 7 , se muestran las mediciones obtenidas, del banco de ensayos motor, en relación al gasto másico del combustible. Este gasto másico es el que se necesita para que el motor térmico opere a una condición de régimen de giro específica, considerando la carga que le imprime el freno motor.

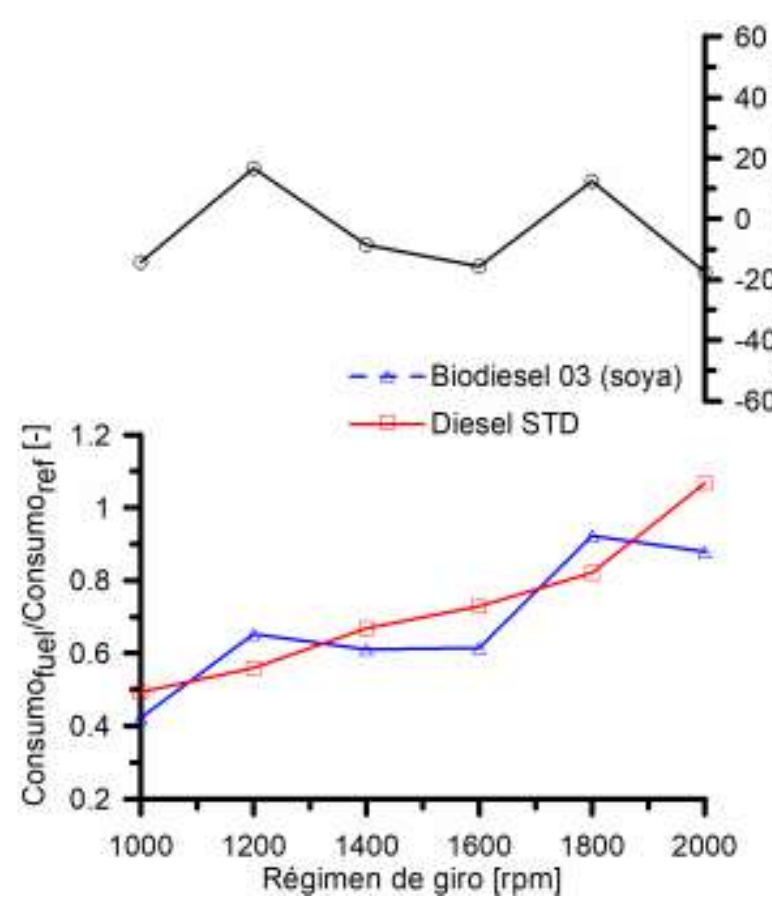

Figura 7 Resultados del banco de ensayos motor. Abajo: gasto másico adimensional de combustible frente al régimen de giro motor; Arriba: la diferencia porcentual del gasto másico del combustible del motor térmico usando diésel estándar y el biocombustible generado a partir de aceite de soya

En esta figura 7, en la parte de abajo, se muestra el consumo de combustible en su forma adimensional, que nuevamente, es de gran utilidad cuando se desea contrastar diferencias a efectos comparativos. Con las diferentes condiciones de operación ensayadas; donde se ha usado una condición real de operación registrada como referencia. 
En esta figura 7 se muestra una diferencia de $16.6 \%$ de mayor consumo, a $1200 \mathrm{rpm}$, cuando se usa el biocombustible obtenido de aceite de soya frente al diésel de uso comercial. No obstante, a $2000 \mathrm{rpm}$, se muestra una diferencia de $17.5 \%$ de menor consumo cuando se usa el biodiésel generado con aceite de soya frente al diésel estándar. Estas diferencias se deben, por una parte, a las características fisicoquímicas de densidad, viscosidad, poder calorífico del combustible y, por otra parte, a la forma en que se lleva a cabo el proceso de combustión, durante la carrera de expansión del motor de combustión interna presentado en la figura 3. Por otra parte, el rendimiento térmico del motor de combustión interna es un parámetro que evalúa la tasa de energía suministrada por el combustible frente a la potencia que entrega el motor térmico al freno-motor. El rendimiento térmico contiene información del poder calorífico del combustible. De esta forma, en la figura 8 se muestra el rendimiento térmico estimado con los resultados obtenidos en el banco de pruebas motor.

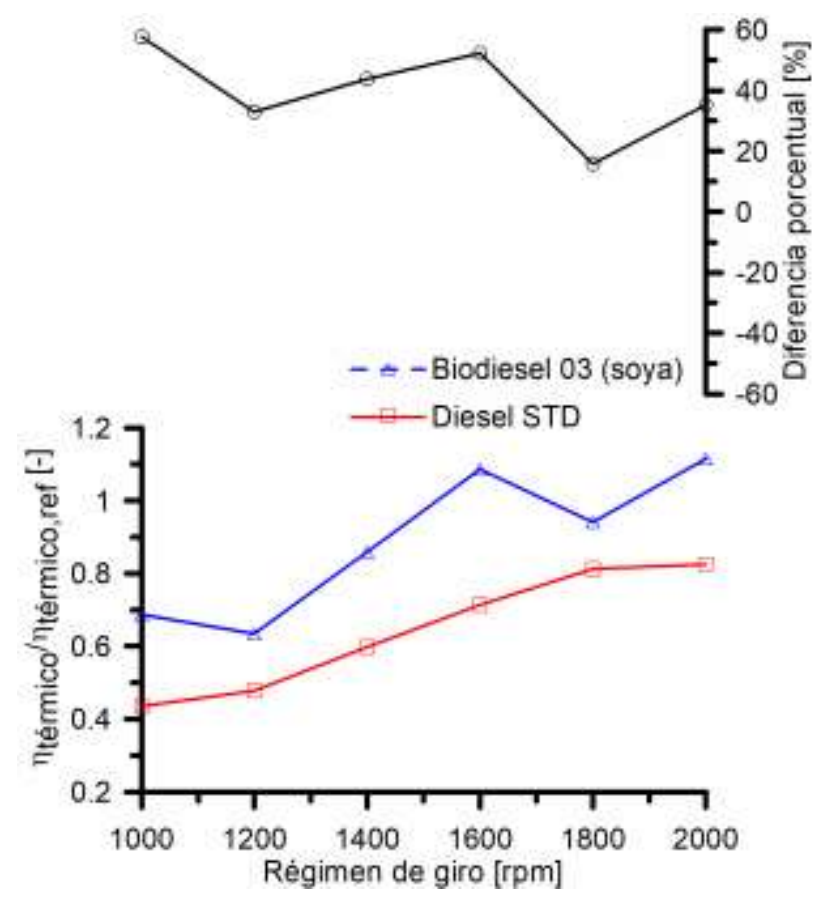

Figura 8 Resultados del banco de ensayos motor. Abajo: rendimiento térmico adimensional frente al régimen de giro motor; Arriba: la diferencia porcentual del rendimiento térmico del motor de combustión interna usando diésel estándar y el biocombustible generado a partir de aceite de soya

En esta figura 8, en la parte de abajo, se muestra el rendimiento térmico del motor en su forma adimensional, donde se ha usado una condición real de operación registrada como referencia.
En esta figura 8 se muestra una diferencia de $33 \%$ de mayor eficiencia térmica, a 1200 rpm, cuando se usa el biocombustible obtenido de aceite de soya frente al diésel de uso comercial. También, a 2000 rpm, se muestra una diferencia de $35 \%$ de mayor eficiencia térmica, cuando se usa el biodiésel generado con aceite de soya frente al diésel estándar. Cabe mencionar, que para la obtención del rendimiento térmico del motor se ha usado para ambos combustibles, que se muestran en la figura 8, como referencia, el valor de poder calorífico del diésel comercial. De esta forma, es evidente que para una potencia y consumo de combustible fija, el incrementar el valor de poder calorífico provoca que el rendimiento térmico del motor se reduzca.

No obstante, la mejora en la potencia lograda con el biocombustible, como se muestra en la figura 6, indican que el biodiésel obtenido, a partir de aceite de soya, tienen mejores características fisicoquímicas, por ende, se puede llevar a cabo una mejor eficiencia de combustión en el interior del motor de combustión interna alternativo.

Por otra parte, a partir de aquí se muestran los resultados de obtenidos con el biocombustible generado con aceite de canola. De esta forma, en la figura 9 se muestra la potencia obtenida con el diésel comercial frente al biodiésel obtenido con aceite de canola.

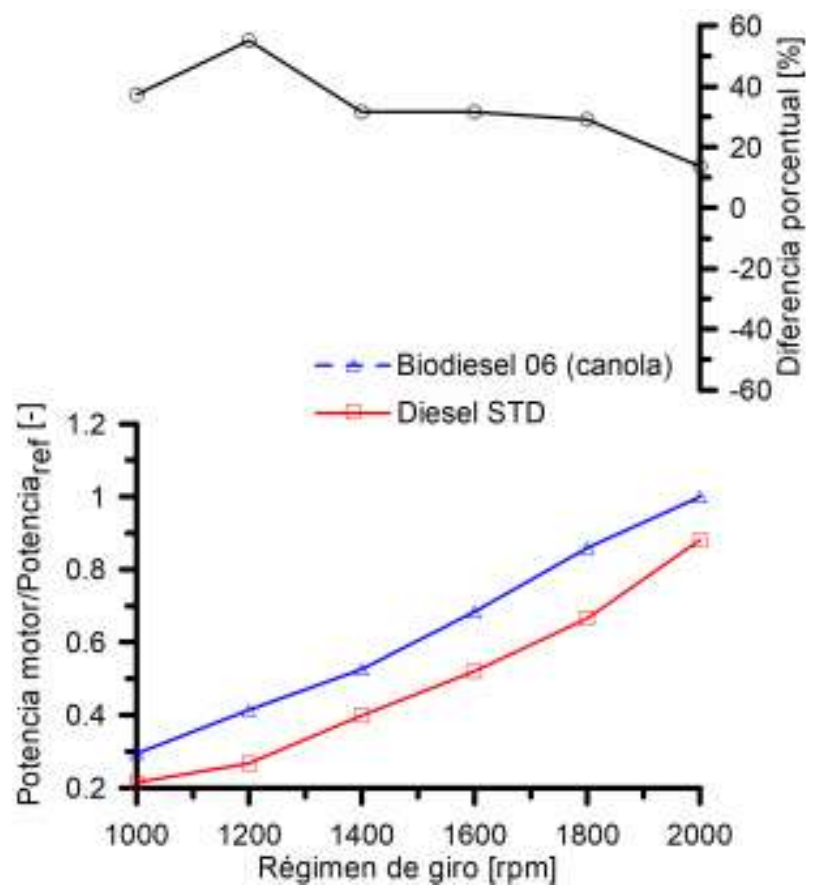

Figura 9 Resultados del banco de ensayos motor. Abajo: potencia adimensional frente al régimen de giro motor; Arriba: la diferencia porcentual de la potencia del motor térmico usando diésel estándar y el biocombustible generado a partir de aceite de canola

JIMÉNEZ-MACEDO, Víctor Daniel, VILLAGÓMEZ-GALINDO, Miguel y DE LA GARZA-DE LEÓN, Oscar Alejandro. Análisis de las prestaciones de un motor de combustión interna alternativo usando biocombustibles: (i) aceite de soya; (ii) aceite de canola. Revista de Energías Renovables. 2020 
En la figura 9 se muestra la potencia obtenida del banco de pruebas motor. Los resultados de potencia se muestran en su forma adimensional. Se ha usado la misma condición de referencia que fue empleada en los resultados de la figura 6. Así pues, se muestra que la potencia registrada, cuando se usa el biocombustible a partir de aceite vegetal de canola es mayor en todas las condiciones de operación. De esta forma, los resultados de diferencia porcentual, se muestran en la parte de arriba de esta misma figura 9. Que indican una diferencia máxima, nuevamente, de $55 \%$ cuando el tacómetro marca $1200 \mathrm{rpm} \mathrm{y}$; una diferencia mínima de $13.6 \%$ para un régimen de giro de $2000 \mathrm{rpm}$

Por otra parte, los resultados obtenidos del gasto másico del combustible, usando el bicombustible a partir de aceite de canola, se muestran en la figura 10 .

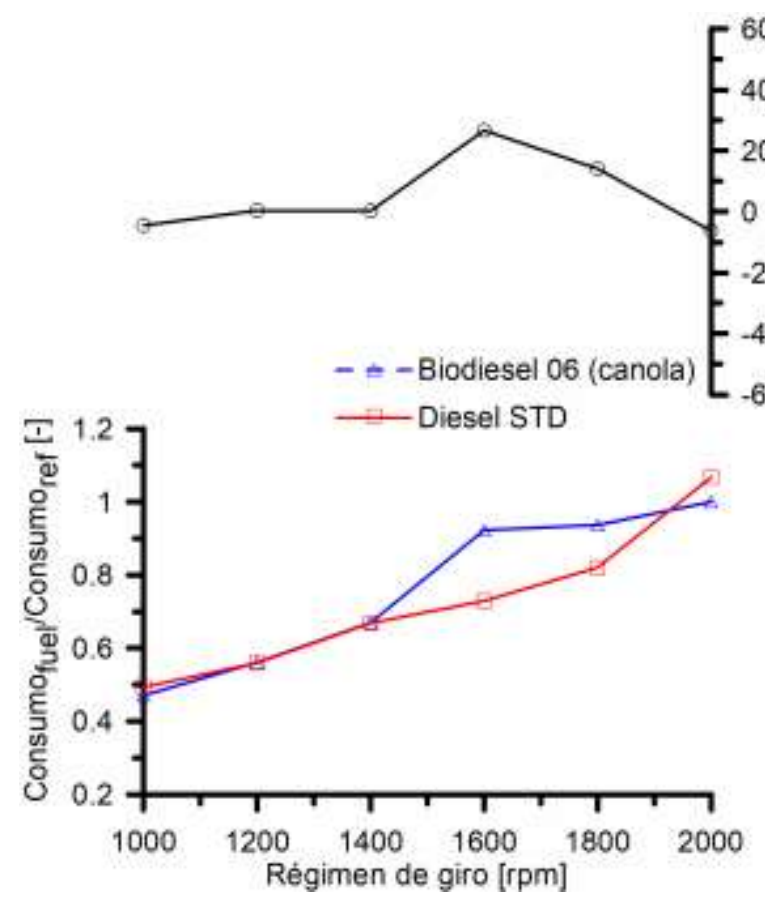

Figura 10 Resultados del banco de ensayos motor. Abajo: gasto másico adimensional de combustible frente al régimen de giro motor; Arriba: la diferencia porcentual del gasto másico del combustible del motor térmico usando diésel estándar y el biocombustible generado a partir de aceite de canola

El gasto másico que se muestra, en la parte de abajo, de la figura 10, está en su forma adimensional, donde se ha usado la misma condición de referencia usada en la figura 7. Así pues, ahora en la parte de debajo de la figura 10 se muestra una diferencia de $0.38 \%$ de mayor consumo, a $1200 \mathrm{rpm}$, cuando se usa el biocombustible obtenido de aceite de canola frente al diésel de uso común.
No obstante, se muestra una diferencia de $6.25 \%$ de menor consumo, cuando se usa el biodiésel generado con aceite de canola frente al diésel estándar, a 2000 rpm.

Por otra parte, en la figura 11, se muestra el rendimiento térmico del motor de combustión interna, que se ha obtenido con los resultados obtenidos en el banco de ensayos motor.

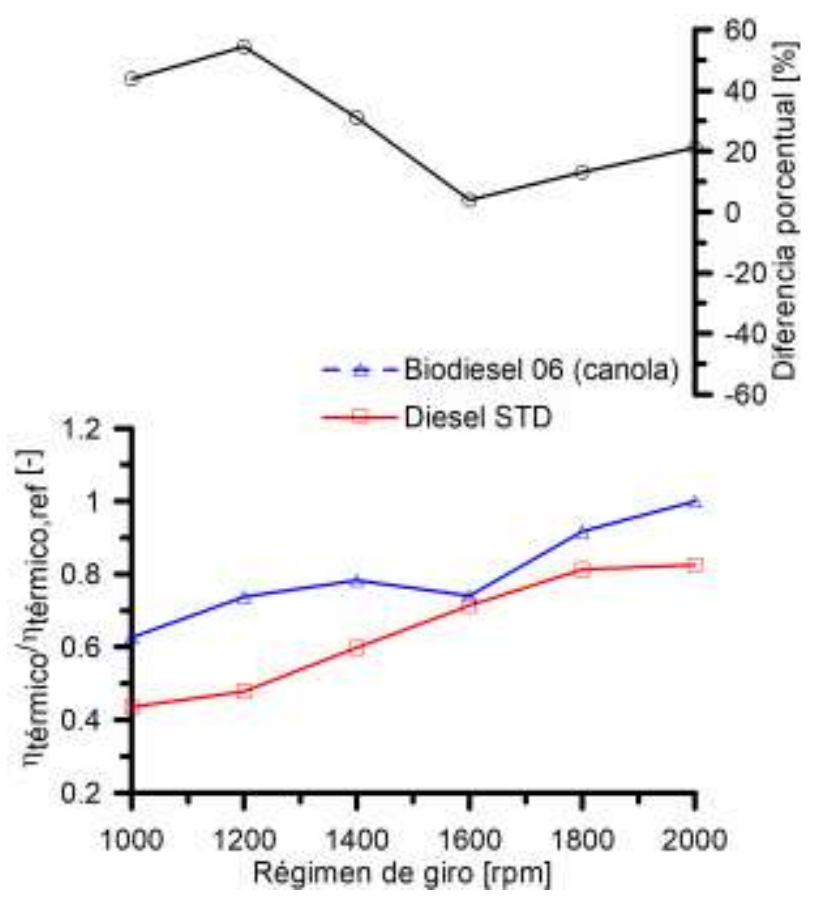

Figura 11 Resultados del banco de ensayos motor. Abajo: rendimiento térmico adimensional frente al régimen de giro motor; Arriba: la diferencia porcentual del rendimiento térmico del motor de combustión interna usando diésel estándar y el biocombustible generado a partir de aceite de canola

Los resultados de la parte de debajo de esta misma figura se muestran en su forma adimensional, donde fue usada la misma condición de referencia empleada en la figura 8 . En esta figura 11 se muestra una diferencia de 54 $\%$, de mayor eficiencia térmica, a $1200 \mathrm{rpm}$, cuando se usa el biodiésel generado de aceite de canola frente al diésel de uso común.

Esta misma tendencia, se muestra a 2000 rpm, donde hay una diferencia de $21 \%$ de mayor eficiencia térmica cuando se usa el biodiésel generado con aceite de canola frente al diésel estándar. También, para el presente caso, para la obtención del rendimiento térmico del motor, se ha usado para ambos combustibles, que se muestran en la figura 11, como referencia el valor de poder calorífico del diésel comercial. 
Pero, la mejora en la potencia motor, cuando se emplea el biocombustible de aceite de canola, es un indicativo que está relacionado con las características fisicoquímicas, y que se ven reflejadas en el proceso de combustión y en el funcionamiento general del motor de combustión interna alternativo.

Por otra parte, ahora se quiere mostrar, la temperatura del gas de escape. Que es una información de utilidad si se quieren realizar estudios de transferencia de calor, no obstante, ese estudio queda fuera del alcance del presente trabajo de investigación. De esta forma, en la figura 12 se muestra la temperatura del gas del escape que se ha obtenido con las mediciones registradas en el banco motor.

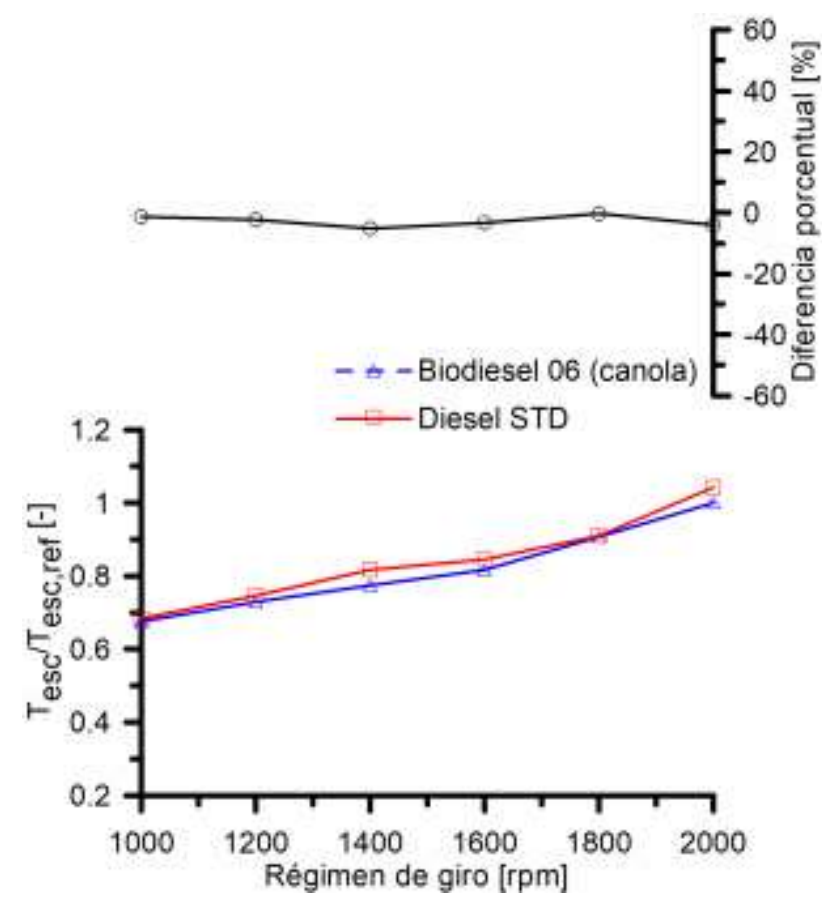

Figura 12 Resultados del banco de ensayos motor. Abajo: Temperatura de escape adimensional frente al régimen de giro motor; Arriba: la diferencia porcentual de la temperatura de escape del motor de combustión interna usando diésel estándar y el biocombustible generado a partir de aceite de canola

En la parte de debajo de la figura 12 se muestra la temperatura en el sistema de escape obtenida en el banco motor. Los resultados de temperatura, en esta figura, se muestra en su forma adimensional, donde fue usada la misma medición experimental de referencia de las anteriores figuras. Así pues, los datos de temperatura muestran diferencias inferiores al 5 $\%$ entre ambos combustibles. Donde en todas las condiciones de régimen de giro, la temperatura del gas de escape fue ligeramente mayor cuando se usa el diésel de origen fósil frente al biodiésel obtenido con aceite de canola.

\section{Conclusiones}

En el presente trabajo se realiza una evaluación de dos biocombustibles frente al diésel de origen fósil. De esta forma, primeramente, se ha producido los dos biocombustibles, a partir de aceite de soya y aceite de canola. Seguidamente, se ha descrito los recursos experimentales, que incluyen al motor de combustión interna alternativo en estudio y el banco de ensayos motor. Que incorpora un freno motor para de medir la potencia generada por el motor térmico en estudio.

En la siguiente sección, se muestran los resultados registrados de forma experimental que incluye: (i) la potencia-motor; (ii) el consumo de combustible; (iii) rendimiento térmico y; (iv) temperatura del gas de escape.

Estos resultados indican diferencias porcentuales promedio de $32 \%$ y $33 \%$ en la potencia motor, cuando se usa el biocombustible generado a partir de aceite de soya y aceite de canola respectivamente; frente al diésel estándar. Por otra parte, en cuanto al gasto másico se observaron porcentuales promedios de $-4.5 \mathrm{y}$ $5.12 \%$. Asimismo, en los resultados estimados del rendimiento térmico las diferencias porcentuales promedio fueron de 39.6 y $27.9 \%$, también, estos valores corresponden, cuando se usa el biocombustible elaborado a partir de aceite de soya y aceite de canola respectivamente; frente al diésel comercial. Por otra parte, en cuanto a los resultados registrados de temperaturas del gas de escape, estas fueron menores al $5 \%$. En la cual, en todas las condiciones de régimen de giro, las temperaturas fueron mayores cuando se usa el diésel de origen fósil frente al biodiésel obtenido con aceite de canola. Finalmente, se puede indicar que las diferencias obtenidas, en los diferentes parámetros del motor de combustión interna alternativo, se deben a las características fisicoquímicas de los tres diferentes combustibles usados.

\section{Agradecimientos}

Se agradece al Programa para el Desarrollo Profesional Docente, para el Tipo Superior, PRODEP, por el apoyo recibido, así mismo se agradece de igual forma al CONACYT y a la Coordinación de la Investigación Científica de la Universidad Michoacana de San Nicolás de Hidalgo CIC-UMSNH. 


\section{Referencias}

Blair G.P. (1996) Design and simulation of twostroke engines.

García Martínez, E. (2020). Elaboración de modelos de motor numéricos para formación en la puesta a punto de motor. TFG. UPV, Valencia, España.

Gerhard Knothe, Jürgen Krahl, Jon Van Gerpen (2010). The Biodiesel Handbook (Second Edition). AOCS Press, ISBN 9781893997622. https://doi.org/10.1016/B978-1-893997-622.50003-6.

Heywood J. B. (1988). Internal combustion engine fundamentals. McGraw-Hill Publishing.

Jiménez Macedo et al. (2017). Evaluación de una mezcla de combustible hidrógeno y gasolina para su uso en motores de combustión interna: emisiones contaminantes. Academia Journals, Vol. 9, No. 6, ISSN 1946-5351, Celaya, México.

Jiménez Macedo et al. (2019). Construcción, optimización y evaluación experimental de una celda de hidrógeno. CECTI, Morelia, México.

Jiménez Macedo et. al. (2018) Transferencia de calor local en un sistema de escape en un motor de $4.1 \mathrm{~L}$ de encendido por chispa. Academia Journals, Vol. 10, No. 3, ISSN 1946-5351, Morelia, México.

Mofijur, M., Rasul, M. G., Hyde, J., Azad, A. K., Mamat, R., \& Bhuiya, M. M. K. . (2016). Role of biofuel and their binary (diesel-biodiesel) and ternary (ethanol-biodiesel-diesel) blends on internal combustion engines emission reduction. Renewable and Sustainable Energy Reviews, 53, 265-278.

Natkin RJ, Tang X, Whipple KM, Kabat DM. (2002). Stockhausen WF. Ford hydrogen engine laboratory testing facility. SAE Technical Paper No. 2002-01-0241.

Payri F. y Desantes J.M. (2011) Motores de combustión interna alternativos. UPV, Valencia, España.

Peyton, K. (2016). Nalco Champion fuel field manual (3a edición). EE. UU.: McGraw-Hill.
Rakopoulos Constantine et Giakoumis Evangelos. (2009). Diesel Engine Transient operation, Springer-Verlag London Limited, ISBN 978-1-84882-374-7, DOI 10.1007/978-184882-375-4.

Stikhun, A. (2020). Análisis numérico de un regenerador de porosidad variable y su influencia en el rendimiento de un motor Stirling. PFC. Universidad de Málaga, España.

JIMÉNEZ-MACEDO, Víctor Daniel, VILLAGÓMEZ-GALINDO, Miguel y DE LA GARZA-DE LEÓN, Oscar Alejandro. Análisis de las prestaciones de un motor de combustión interna alternativo usando biocombustibles: (i) aceite de soya; (ii) aceite de canola. Revista de Energías Renovables. 2020 